\title{
ON THE SEPARATION OF THE PLANE BY A CONTINUUM*
}

BY R. L. MOORE

In his paper, On the separation of the plane by irreducible continua, $\dagger \mathrm{W}$. A. Wilson obtains the following theorem. Let $F$ be the union of two bounded continua $H_{1}$ and $H_{2}$ having these properties: $H_{1}$ and $H_{2}$ are irreducible about $A+B$; $H_{1} \cdot H_{2}=A+B$ where $A$ and $B$ are continua and $A \cdot B=0$; $H_{1}$ and $H_{2}$ contain subcontinua $C_{1}$ and $C_{2}$ respectively such that $\alpha=C_{1} \cdot C_{2} \cdot A \neq 0, \beta=C_{1} \cdot C_{2} \cdot B \neq 0, C_{1}$ and $C_{2}$ are irreducible between $\alpha$ and $\beta$ and $F=C_{1}+C_{2}$. Then $F$ cuts the plane and is the frontier of exactly two components of its complement.

In the present paper I will establish two theorems which together yield more information than Wilson's theorem.

Theorem 1. If $A$ and $B$ are two mutually exclusive continua and the bounded continua $H_{1}$ and $H_{2}$ are both irreducible about $\ddagger A+B$, and $H_{1} \cdot H_{2}=A+B$, then $H_{1}+H_{2}$ is not the boundary of more than two domains.

Proof. Let $F$ denote the point set $H_{1}+H_{2}$. With the aid of the fact that $H_{1}-(A+B)$ and $H_{2}-(A+B)$ are $\S$ connected, it may be easily seen that there exists an inversion of the plane about some point of the complement of $F$ such that if, for each point set $M, \bar{M}$ denotes the image of $M$ under this inversion, then no bounded complementary domain of $\bar{A}$ or $\bar{B}$ contains a point of $\bar{F}$. If, to each of the continua $\bar{A}$ and $\bar{B}$, all its bounded complementary domains are added and

* Presented to the Society, December 28, 1927.

$\dagger$ This Bulletin, vol. 33 (1927), pp. 733-744.

$\ddagger$ If $A$ is a closed subset of a continuum $C$ and no proper subcontinuum of $C$ contains $A$ then $C$ is said to be irreducible about $A$. See W. A. Wilson, loc. cit., Definition I.

$\S$ See W. A. Wilson, loc. cit., Lemma I. 
the continuum so obtained is regarded as an element and each point that does not belong to $A$ or to $B$ is regarded as an element, the collection $G$ of elements so obtained is an upper semi-continuous* collection. Furthermore, no element of the collection $G$ separates the plane and together they fill up the plane. It follows that, if the elements of $G$ are regarded as points, all theorems of the analysis situs of the plane hold true, in the space so obtained, in the sense described, in detail, in my paper Concerning upper semicontinuous collections of continua. $\dagger$ Hence, by a theorem used in a similar connection in the proof of Theorem 2, there exists a simple closed curve $J$ of elements of $G$ which separates $\bar{H}_{1}-(\bar{A}+\bar{B})$ from $\bar{H}_{2}-(\bar{A}+\bar{B})$. If, for each value of $i$ $(i=1,2), t_{i}{ }^{\prime}$ denotes the segment of $J$ whose extremities are the elements of $G$ that contain $\bar{H}_{1}$ and $\bar{H}_{2}$, respectively, and $D_{i}$ denotes the complementary domain of $\bar{F}$ that contains $t_{i}$, then, if $\bar{F}$ is the boundary of a domain, that domain must contain a point of $t_{1}$ or of $t_{2}$, and therefore must be identical with $D_{1}$ or with $D_{2}$.

TheOREM 2. If $\alpha$ and $\beta$ are two mutually exclusive closed point sets and each of the bounded continua $C_{1}$ and $C_{2}$ is irreducible from $\ddagger \alpha$ to $\beta$, and $C_{1} \cdot C_{2}=\alpha+\beta$, then $C_{1}+C_{2}$ is the boundary of at least two distinct domains.

Proof. Let $F$ denote the point set $C_{1}+C_{2}$. With the help of the fact that $C_{1}-(\alpha+\beta)$ and $C_{2}-(\alpha+\beta)$ are $\S$ connected

* See my paper, Concerning upper semi-continuous collections of continua which do not separate a given continuum, Proceedings of the National Academy, vol. 10 (1924), pp. 356-360.

$\dagger$ Transactions of this Society, vol. 27 (1925), pp. 416-428. In the first line of the statement of Theorem 24 on page 427 of this paper, the word "bounded" should be inserted between the words "closed" and "point".

$\ddagger$ If $K$ and $L$ are two closed and mutually exclusive point sets, the continuum $H$ is said to be irreducible from $K$ to $L$ if it contains at least one point of $K$ and at least one point of $L$ but contains no proper subcontinuum that does so. See Anna M. Mullikin, Certain theorems relating to plane connected sets, Transactions of this Society, vol. 24 (1922), pp. 144-162. Wilson uses the term irreducible between instead of irreducible from.

$\S$ See Anna M. Mullikin, loc. cit. 
it may be shown that there exists an inversion of the plane, about some point of the complement of $F$, such that, using the notation indicated in the above proof of Theorem 1, no bounded complementary domain of any maximal connected subset of $\bar{\alpha}+\bar{\beta}$ contains any point of $\bar{F}$. To each maximal connected subset $X$ of $\bar{\alpha}$ add all the bounded complementary domains of $X$ and call each point set so obtained an element. Let $G_{\alpha}$ denote the set of all such elements. Let $G_{\beta}$ denote a set of elements determined in the same way from the maximal connected subsets of $\bar{\beta}$. Let $G$ denote the upper semicontinuous collection whose elements are the elements of $G_{\alpha}$, the elements of $G_{\beta}$, and the points that belong to no element of $G_{\alpha}$ or $G_{\beta}$. Let $H_{1}$ denote the set of elements $\bar{C}_{1}-(\bar{\alpha}+\bar{\beta})+G_{\alpha}+G_{\beta}$ and let $H_{2}$ denote the set $\bar{C}_{2}-(\bar{\alpha}+\bar{\beta})$ $+G_{\alpha}+G_{\beta}$. The set of elements $G_{\alpha}+G_{\beta}$ is a totally disconnected set of elements and it consists of all elements common to the two closed, connected and bounded sets $H_{1}$ and $H_{2}$. Let $Q$ denote a simple closed curve enclosing $H_{1}+H_{2}$. There exists an arc $A B$ such that (1) $A$ belongs to $Q$, (2) $B$ belongs to $\bar{C}_{1}-(\bar{\alpha}+\bar{\beta})$ or to $\bar{C}_{2}-(\bar{\alpha}+\bar{\beta})$ and (3) $B$ is the only element of $A B$ that belongs to $H_{1}+H_{2}$. Suppose $B$ belongs to $\bar{C}_{1}-(\bar{\alpha}+\bar{\beta})$. Then $\bar{C}_{2}-(\bar{\alpha}+\bar{\beta})$ lies in a bounded complementary domain of $Q+A B+H_{1}$. Call this domain $D$. There exists* a simple closed curve $J$ of elements of $G$ such that (1) $J$ contains at least one element of $G_{\alpha}+G_{\beta}$ and encloses at least one element of $\bar{C}_{2}-(\bar{\alpha}+\bar{\beta}),(2)\left(J+G_{\alpha}+G_{\beta}\right)$ $-\left(G_{\alpha}+G_{\beta}\right)$ is a subset of $D$ and it contains no element of $\bar{C}_{2}-(\bar{\alpha}+\bar{\beta})$. With the help of the fact that $\bar{C}_{2}-(\bar{\alpha}+\bar{\beta})$ is connected and that every element of $G_{\alpha}+G_{\beta}$ is a limit element of $\bar{C}_{2}-(\bar{\alpha}+\bar{\beta})$ it easily follows that $J$ contains $G_{\alpha}+G_{\beta}$ and encloses the whole of $\bar{C}_{2}-(\bar{\alpha}+\bar{\beta})$. Furthermore, $\bar{C}_{1}-(\bar{\alpha}+\bar{\beta})$ is wholly without $J$. It is easy to see that the curve $J$ contains two segments $t_{1}$ and $t_{2}$ such that (1) for each $i(i=1,2) t_{i}$ contains no element of $G_{\alpha}$ or of $G_{\beta}$ but the end elements of $t_{i}$ belong to $G_{\alpha}$ and to $G_{\beta}$ respectively and

* See this Bulletin, vol. 33 (1927), p. 521, Abstract 38. 
(2) $t_{1}$ and $t_{2}$ belong to different complementary domains of $H_{1}+H_{2}$. The segments $t_{1}$ and $t_{2}$ are point sets in the ordinary sense* and they are connected subsets of the complement (in the ordinary sense) of $\bar{F}$ and each of the sets $\bar{\alpha}$ and $\bar{\beta}$ contains at least one limit point of $t_{1}$ and at least one limit point of $t_{2}$. In the space of the ordinary points of the plane, for each $i$ let $D_{i}$ denote the complementary domain of $\bar{F}$ that contains $t_{i}$. The continuum $\bar{F}$ is the boundary both of $D_{1}$ and of $D_{2}$. For let $N$ denote the boundary of $D_{1}$. If $N$ does not contain $\bar{C}_{2}$ then $N \cdot \bar{C}_{2}$ contains no connected subset containing both a point of some element of $G_{1}$ and a point of some element of $G_{2}$. Hence there exists an $\operatorname{arc} A_{1} A_{2}$ from a point $A_{1}$ of $t_{1}$ to a point $A_{2}$ of $t_{2}$ and lying, except for these points, wholly in the interior of $J$ and having no point in common with $N \cdot \bar{C}_{2}$. Since $\bar{C}_{1}-(\bar{\alpha}+\bar{\beta})$ lies wholly without $J$ it has no point in common with $A_{1} A_{2}$. Thus $A_{1} A_{2}$ has no point in common with $N$. But $A_{1}$ and $A_{2}$ belong to different complementary domains of $N$. Thus the supposition that $N$ does not contain $\bar{C}_{2}$ has led to a contradiction. In a similar way it may be shown that $N$ contains $\bar{C}_{1}$. Therefore $\bar{F}$ is the boundary of $D_{1}$. By a similar argument it may be shown to be the boundary of $D_{2}$. It follows that $F$ is the boundary of at least two domains.

The University of TeXas

* This does not imply that they are necessarily subsets of arcs whose elements are all points in the ordinary sense. It is possible, for example, that $t_{1}$ may be the set of all points of the graph of $y=\sin (1 / x)$ that lie between the lines $x=0$ and $x=1$. This point set is not a subset of any arc whose elements are all points but it is a segment of an arc of which the only element which is not a point is the straight line interval from $(0,1)$ to $(0,-1)$. 